\title{
DESENVOL VIMENTO E INTEGRAÇÃO DE UM SIMULADOR DE FLUXO SUBTERRÂNEO A UM SISTEMA DE INFORMAÇÕES GEOGRÁFICAS
}

\section{DEVELOPMENT AND INTEGRATION OF A GROUNDWATER MODEL TO A GEOGRAPHIC INFORMATION SYSTEM}

\author{
Cristiano das Neves Almeida ${ }^{1}$, Jackson Roehrig ${ }^{2}$ e Edson Cezar Wendland ${ }^{3}$
}

Recebido em: 24/01/2006; aceito em: 21/09/2006

\begin{abstract}
RESUMO Apresenta-se neste artigo a implementação de uma parte do Sistema de Suporte à Decisão Espacial (SSDE) desenvolvido para a área de recursos hídricos, mais especificamente no que tange à área de águas subterrâneas. Este sistema, denominado ARENA (Análise de Recursos Naturais), é formado por um simulador do fluxo de águas subterrâneas, um Sistema de Informações Geográficas aberto de código livre, o JUMP, um banco de dados georeferenciado e as GUI's (Graphical User Interface) de acesso aos dados. O Método de Elementos Finitos (MEF) foi utilizado para resolver as equações diferenciais que descrevem o fluxo de águas subterrâneas. O SSD desenvolvido através da Programação Orientada a Objetos (POO) representa os sistemas por meio de classes. Uma contribuição fundamental é a integração do simulador ao SIG, uma vez ambos utilizam as mesmas entidades geométricas no processo de modelagem. Ao final, apresentam-se aplicações do ARENA a um sistema hipotético (em transiente) a fim de avaliar as potencialidades do SSD, onde se pode ver que as ferramentas do JUMP podem ser utilizadas para apoiar o processo de simulação do fluxo de águas subterrâneas, apesar de que algumas limitações foram detectadas.
\end{abstract}

Palavras-chave - fluxo de águas subterrâneas, modelagem, Sistema de Informação Geográfica, Sistemas de Suporte à Decisão Espacial, Programação Orientada a Objetos.

\begin{abstract}
This paper shows the implementation of a section of a Spatial Decision Support System (SDSS) which was developed to the water resources area, more specifically to the groundwater area. The DSS, called ARENA (Análise de Recursos Naturais, in Portuguese), is made up of a groundwater model, a Geographic Information System (GIS), the JUMP, a georeferenced database and GUIs (Graphical User Interface) to access data. The Finite Element Method (FEM) was used to solve the differential partial equations that governs the groundwater flow. The DSS was developed through Oriented Object (OO) that represents systems based on classes. One important contribution is the integration of the model and the GIS, because both of them use the same geometric entity during the simulation. In the end, applications of the ARENA are presented to a hypothetical system (a non-steady simulation) in order to evaluate the DSS potentialities, where the JUMP tools supported well the groundwater simulation process, even though some limitation were found.
\end{abstract}

Keywords - groundwater flow, model, Geographic Information System, Spatial Decision Support System, Oriented Object.

\section{INTRODUÇÃO}

Tratar de questões de planejamento e gerenciamento de recursos hídricos significa utilizar alguma das ferramentas do trinômio SSD, simuladores e SIG, seja para a simples realização de modelagens com os simuladores (CAVALCANTI, 2002), ou uso de um SIG para análises espaciais e suporte a simulações, (ALMEIDA, 2001; ASSIS, 1999; TSOU e WHITTEMORE, 2001), ou mesmo, o tratamento de sistemas mais complexos com os SSD's, (RODRIGUES, 2005; PORTO e AZEVEDO, 1997).

A partir de 1997, questões de planejamento e gerenciamento de recursos hídricos vêm notadamente ganhando espaço no cenário nacional. A promulgação da Política Nacional de Recursos Hídricos, Lei 9.433, em 1997, pode ser considerada o marco inicial. Cinco ferramentas encontram-se previstas nessa Política, a saber: Os planos de recursos hídricos; o enquadramento dos corpos de água em classe; a outorga de direito de uso dos recursos hídricos; a cobrança pelo uso dos recursos hídricos; e, o sistema de informações sobre recursos hídricos. Esse sistema tem como objetivo principal a coleta, a consistência e a disponibilização de informações sobre as questões quali-quantitativas sobre recursos hídricos no Brasil.

Dentro do contexto dos sistemas de informações encontram-se os Sistemas de Suporte à Decisão (SSD). Aplicações dos SSD's na área de recursos hídricos vêm aumentando, em função das ferramentas disponíveis nesses sistemas. As ferramentas dos SSD's objetivam sistematizar informações (mapas, dados tabulares, séries temporais, etc.) no tempo e no espaço, a fim de se facilitar o entendimento dos sistemas de recursos hídricos.

\footnotetext{
${ }^{1}$ Doutorando da Escola de Engenharia de São Carlos-Universidade de São Paulo (EESC/USP) (almeida74br@yahoo.com.br)

${ }^{2}$ Prof. Doutor do Instituto de Tecnologias para os Trópicos e Sub-Trópicos-Universidade de Ciências Aplicadas de Colônia (Jackson.Roehrig@dvz.fh-koeln.de)

${ }^{3}$ Prof. Doutor da Escola de Engenharia de São Carlos-Universidade de São Paulo (EESC/USP) (ew@sc.usp.br)
} 
Um dos componentes dos SSD's são os modelos. No caso específico de águas subterrâneas, esses modelos são utilizados para resolver equações diferenciais e representar, por exemplo, a variação das cargas hidráulicas nos aqüíferos. Os modelos matemáticos computacionais são extremamente úteis para realizar análises complexas dos aqüíferos e expandir a capacidade do hidrólogo e hidrogeólogo de entender e gerenciar os recursos hídricos subterrâneos. O uso de modelos ajuda a sistematizar as informações de campo e identificar áreas onde informações complementares são necessárias. Nessa área, vários modelos podem ser encontrados, sendo que, em geral, utilizam o Método de Diferenças Finitas ou o Método de Elementos Finitos para resolver as equações diferenciais.

Nos últimos dez anos, com o avanço da Tecnologia de Informação (TI), houve um grande avanço no que diz respeito à área de informática e recursos hídricos. Simuladores como o MODFLOW (MCDONALD, 1988) e o FEFLOW (DIERSCH, 1996), que durante muito tempo foram executados em sistema DOS, passaram a ser executados em sistema Windows $®$, facilitando assim a difusão dos mesmos. Sistemas de Informações Geográficas (SIG's) passaram a compor os SSD's ou e a base de entrada de dados para os programas citados. Já nos últimos cinco anos, tecnologias como a Programação Orientada a Objetos (POO), o uso de formatos como o XML (Extensible Markup Language) e o UML (Unified Modeling Language) têm melhorado a representação e o entendimento de sistemas complexos de recursos hídricos. Essas novas tecnologias procuram representar entidades existentes de forma semelhante à realidade, isto feito por meio de classes, diagramas e formatos padronizados, facilitando assim, a representação, o armazenamento, a manipulação e a troca de dados entre sistemas especialistas.

Dentro do contexto descrito, este artigo trata do desenvolvimento um SSD denominado ARENA (Análise de Recursos Naturais) e apresenta a utilização de novos conceitos de TI para o desenvolvimento desse sistema. O ARENA é composto por simuladores de sistemas de recursos hídricos, porém este artigo trata apenas do simulador do fluxo de águas subterrâneas. Este simulador utiliza o Método de Elementos Finitos para resolver as equações diferenciais parciais, que regem o movimento d'água em meios porosos saturados. Compõe ainda o ARENA um SIG, uma base de dados georeferenciada e as GUI's (Graphical User
Interface), que são os módulos de diálogo e acesso a dados. Inicialmente, apresenta-se a fundamentação teórica que embasou e motivou o desenvolvimento do trabalho. Em seguida, as equações do fluxo de águas subterrâneas e o Método de Elementos Finitos (MEF) são descritas. A implementação computacional do simulador é, então, detalhada. Para tanto, faz-se uso da linguagem UML. Apresenta-se então a integração do simulador ao SIG, formando o SSD. Uma aplicação do ARENA é realizada para um sistema hipotético. Por fim, são apresentadas as conclusões, onde se destacam as ferramentas de análise e tratamento de dados espaciais no apoio ao processo de simulação do fluxo de águas subterrâneas.

\section{FUNDAMENTAÇÃO TEÓRICA}

Em função dos objetivos deste artigo, procedeu-se uma revisão bibliográfica, a fim de se investigar o uso dos SSD's relacionados aos recursos hídricos, aplicações conjuntas de modelos hidrológicos e SIG's e uso da POO na área de recursos hídricos.

No Brasil, um trabalho pioneiro sobre a concepção dos SSD's foi apresentado por Porto e Azevedo (1997). Nesse documento, a estrutura de um SSD formada por cinco elementos: tomador de decisões, módulo de diálogo, base de dados, base de modelos e base de conhecimento. Segundo esses autores, através dos SSD's, os sistemas podem ser mais bem estruturados e, assim, simplificados, facilitando a tomada de decisão acerca do uso dos recursos hídricos. Outros pesquisadores acrescentam a essa composição os SIG's, a fim de tratar a distribuição espacial, característica intrínseca dos sistemas de recursos hídricos, a exemplo, tem-se Almeida (2001), Assis (1999) e Kyrillos e Cirilo (2000) que apresentaram estruturas de SSD's, integração de SIG's aos SSD e aplicações desses sistemas a bacias hidrográficas.

Cavalcanti (2002) aplicou o MODFLOW na bacia do Biritiba-Mirim, localizada na região do Alto Tietê, no Estado de São Paulo. O objetivo do estudo foi a análise do comportamento do aquífero frente à construção de um reservatório superficial, para determinar quais as influências dessa obra sobre os níveis potenciométricos do aqüífero após o enchimento do reservatório. Nesse estudo, um SIG foi utilizado para auxiliar o processo de formação dos arquivos necessários ao simulador e na fase de pós-processamento dos resultados.

Conforme Tsou e Whittemore (2001), a simulação numérica de sistemas subterrâneos complexos requer o gerenciamento e a análise de 
ma grande quantidade de dados de entrada e saída. Os SIG's, por serem uma plataforma integrada para manipulação, análise e disposição de dados, podem facilitar a compilação de dados necessários, a calibração do modelo e a visualização de parâmetros e resultados da modelagem.

Um grande avanço na área de modelagem de águas subterrâneas é a integração de modelos já bem conhecidos, como o MODFLOW, aos SIG's. De acordo com Tsou e Whittemore (2001), a integração de modelos aos SIG's é classificada em dois tipos: na primeira, cria-se uma interface para conversão dos dados armazenados em camadas do SIG para os arquivos textos, sendo estes arquivos as entradas dos modelos. Executa-se o modelo e utiliza-se novamente a interface para transferência dos arquivos textos, de saída do modelo, para camadas do SIG, onde os resultados da simulação são apresentados. Nessa forma de integração, o SIG assume papel de pré-processador (preparação da entrada dos modelos) e pósprocessador (apresentação dos resultados numéricos dos modelos em forma de camadas do SIG); na segunda forma de integração, as equações do modelo são integradas às entidades geométricas do SIG através de modificações no código fonte do SIG realizada por meio de linguagens de macro, que são ferramentas de programação e automatização de tarefas. Segundo esses pesquisadores, a primeira forma tem sido a mais utilizada no processo de integração de SIG's e modelos. Esses pesquisadores apresentaram uma extensão para simulação do fluxo de águas subterrâneas no ArcView ${ }^{\circledR}$ com o simulador MODFLOW. No trabalho de Tsou e Whittemore (2001), a integração foi implementada através de arquivos de dados. Segundo eles, esse tipo de integração tem como desvantagem a duplicação dos dados existentes no ambiente SIG, para formar os arquivos de entrada do simulador. Porém, apresenta a vantagem de não serem necessárias modificações no código do simulador, a fim de integrá-lo ao SIG, evitando-se assim o surgimento de erros.

Segundo os pesquisadores que utilizaram um SIG para dar apoio ao processo de modelagem, os SIG's podem gerar informações valiosas para o processo de tomada de decisão através da geração de mapas com resultados espacializados e sobreposição a outras informações.

Entre as novas técnicas da TI uma merece destaque: o uso da Programação Orientada a Objetos para o desenvolvimento de programas. Filho e Lanna (2003) apresentaram a aplicação da
Modelagem Orientada a Objetos (MOO) a sistemas de recursos hídricos, em especial o caso da simulação da propagação de vazões ao longo de bacias hidrográficas. Para tanto, uma biblioteca de classes básicas foi concebida. Conforme esses autores, a metodologia da MOO procura representar entidades de sistemas de forma similar à realidade.

Wang et al. (2005) apresentaram a implementação do modelo TOPMODEL (BEVEN e KIRKBY, 1979) através da POO a objetos. Sendo esta nova versão implementada com a linguagem $\mathrm{C}++$. Outro trabalho que utiliza conjuntamente a POO, UML e XML para modelagem de sistemas é apresentado por Babaie e Babaie (2004). Nesse artigo, essas ferramentas são utilizadas para modelar unidades geológicas, sendo a necessidade de armazenamento, troca e disponibilização de dados o motivo que levou ao desenvolvimento do trabalho. Segundo esses autores, a utilização do padrão XML permite o gerenciamento eficiente de dados bem como a troca de informações via WEB. Outro exemplo foi apresentado por Lal et al. (2005) para estudo de escoamentos regionais. Esses pesquisadores utilizaram recentes desenvolvimentos da TI para implementação de um modelo de simulação regional, entre esses, orientação a objetos, XML, SIG e bancos de dados.

Segundo os pesquisadores que utilizaram a POO para implementação de novos programas, a utilização dos mecanismos da POO, tais como a herança e o polimorfismo, são apresentadas como vantagens na administração da complexidade dos sistemas modelados. Além disto, o uso de classes para representação de entidades dos sistemas possibilitam o melhor entendimento de problemas e das relações entre diversos sistemas, já que as classes procuram descrever os sistemas de forma similar à realidade.

\section{METODOLOGIA}

A metodologia utilizada para o desenvolvimento do presente trabalho foi divida em quatro fases: a) estudo das equações diferenciais que regem o fluxo de águas subterrâneas; b) aplicação do MEF para resolução das equações diferenciais; c) utilização da POO para implementação do código computacional do simulador; d) integração do simulador a um SIG. A linguagem UML é utilizada para descrever a arquitetura do aplicativo gerado com a linguagem de programação Java, o qual é composto de classes e pacotes. 


\section{Equações Diferenciais e o Fluxo de Águas Subterrâneas}

O simulador do fluxo de águas subterrâneas foi programado para determinação das variações da carga hidráulica nas direções X e Y, ou seja, para casos bidimensionais (2D), considerando os dois casos seguintes:

- Aquífero confinado em regime permanente;

- Aqüífero confinado em regime transiente;

A determinação das equações diferenciais parciais que regem o comportamento do fluxo subterrâneo para esses dois casos, origina-se da aplicação da equação de Darcy (Equação 3.1) e da equação da continuidade a um volume de controle com dimensões infinitesimais. Para o caso unidimensional a equação de Darcy é escrita como:

Onde:

$$
\mathrm{q}=-\mathrm{K} \cdot(\partial \mathrm{h} / \partial \mathrm{t}) \quad(\text { Equação } 1)
$$

q - fluxo subterrâneo, [L/T];

$\mathbf{K}$ - condutividade hidráulica, [L/T];

h - carga hidráulica, [L];

A aplicação das equações anteriormente mencionadas considerando o regime transiente resulta na equação 3.2 .

$\partial\left(-\mathrm{K}_{\mathrm{x}} \cdot\right.$ b. $\left.\partial \mathrm{h} / \partial \mathrm{x}\right) / \partial \mathrm{x}+\partial\left(-\mathrm{K}_{\mathrm{y}} \cdot \mathrm{b} \cdot \partial \mathrm{h} / \partial \mathrm{y}\right) / \partial \mathrm{y}=\mathrm{W}-\mathrm{S}$. $\partial \mathrm{h} / \partial \mathrm{t}$

(Equação 1)

Onde:

$\mathbf{K}_{\mathbf{x}}$ e $\mathbf{K}_{\mathbf{y}}$ - condutividade hidráulica nas direções $\mathrm{X}$ e $\mathrm{Y},[\mathrm{L} / \mathrm{T}]$;

$\mathbf{S}$ - coeficiente de armazenamento [sem unidade], que no caso de aqüífero confinado é igual ao coeficiente de armazenamento específico $\left(\mathrm{S}_{0}\right)$ multiplicado pela espessura do aqüífero;

W - infiltração ou extração d'água através de poços, ou outras fontes/sumidouros $\left[\mathrm{L}^{3} / \mathrm{T}\right]$;

Caso não haja variações da carga hidráulica ao longo do tempo, o segundo termo do lado direito da equação 2 é anulado, tendo-se assim a equação representativa para aqüíferos confinados em regime permanente (Equação 3).

$$
\mathrm{K}_{\mathrm{x}} \cdot \mathrm{b} \cdot \partial \mathrm{h}^{2} / \partial \mathrm{x}^{2}+\mathrm{K}_{\mathrm{y}} \cdot \mathrm{b} \cdot \partial \mathrm{h}^{2} / \partial \mathrm{y}^{2}=-\mathrm{W} \quad \text { (Equação 3) }
$$

Assim, têm-se as duas equações diferenciais parciais a serem resolvidas pelo MEF.

\section{O Método de Elementos Finitos}

O Método de Elementos Finitos (MEF), diferentemente, do Método das Diferenças Finitas (MDF) vem sendo pouco aplicado na área de recursos hídricos. O principal fator para tal afirmação é o fato de que o MEF requer um embasamento matemático mais elaborado, exigindo um maior esforço do desenvolvedor de modelos quando de sua dedução matemática e implementação computacional. Já o MDF é um dos métodos numéricos mais utilizados, pois a dedução deste método é mais facilmente entendida.

Segundo Chapra e Canale (2002), o funcionamento do MEF podem ser resumidas em seis passos:

1- Discretização do sistema através de elementos triangulares. Neste caso específico foram utilizados elementos triangulares, compostos por três nós;

2- Montagem das matrizes local para cada um desses triângulos. A construção de cada matriz local considera as propriedades hidráulicas do aqüífero, condutividade hidráulica e o coeficiente de armazenamento, e as características geométricas dos triângulos;

3- Construção da matriz representativa do sistema completo. Através das matrizes locais é montada a matriz global, que conterá informações sobre as propriedades hidráulicas do aquíífero e geométricas do conjunto de triângulos. Ao final desta etapa, tem-se um sistema de equações lineares na seguinte forma: $[\mathrm{K}] .\{\mathrm{h}\}=\{\mathrm{Q}\}$, onde $[\mathrm{K}]$ é a matriz global, $\{\mathrm{h}\}$ as cargas hidráulicas nos nós e $\{\mathrm{Q}\}$ as vazões nos nós;

4- Inserção das condições de contorno no sistema de equações formado no item anterior. Três possíveis condições podem ser usadas: cargas hidráulicas conhecidas (condição de Dirichlet); regiões de fluxo conhecido (condição de Neumann); ou uma relação linear entre fluxo e a carga hidráulica (condição de Cauchy). A consideração de alguma dessas condições de contorno modificará o sistema de equações, em geral reduzindo o número de incógnitas;

5- Resolução do sistema de equações, que resultará num vetor com as cargas hidráulicas $\{\mathrm{h}\}$ nos nós dos elementos triangulares;

6- Por fim, são apresentados os resultados, sob forma de mapa com as equipotenciais, por exemplo. Também podem ser calculadas outras variáveis, por exemplo, o fluxo da água subterrânea.

Nessa primeira fase de desenvolvimento do aplicativo o caso dos aqǘferos livres e/ou em regime transiente não foram programados. Para o caso programado, de aquíferos confinados em regime permanente, a aplicação do MEF gera um sistema de equações como o descrito no item III, das etapas de funcionamento do MEF. Para os sistemas em regime transiente, foi aplicado o método de Crank-Nicholson para aproximação no tempo. Assim, foi gerado o seguinte sistema de equações lineares, representado matricialmente: 
$(\mathrm{C} / \Delta \mathrm{t}+\mathrm{K} / 2) \cdot \mathrm{h}(\mathrm{t}+\Delta \mathrm{t})=$

$$
\begin{gathered}
(\mathrm{C} / \Delta \mathrm{t}-\mathrm{K} / 2) \cdot \mathrm{h}(\mathrm{t})+1 / 2 \cdot[\mathrm{Q}(\mathrm{t}+\Delta \mathrm{t})+\mathrm{Q}(\mathrm{t})] \\
(\text { Equação } 4)
\end{gathered}
$$

Onde:

- $\Delta t$ - é o passo de tempo, [T];

- C-é a matriz global, contendo as características geométricas dos elementos triangulares e o coeficiente de armazenamento (S) do sistema;

- $h(t+\Delta t)$ - são as cargas hidráulicas no instante $t+\Delta t,[\mathrm{~L}]$, variáveis desconhecidas do problema;

- $\mathrm{h}(\mathrm{t})$ - cargas hidráulicas no tempo t, [L], conhecidos;

- $Q(t+\Delta t)$ e $\mathrm{Q}(\mathrm{t})$ - são os vetores com as vazões nos nós dos elementos triangulares nos instante $t+\Delta t$ e t, $[\mathrm{L} / \mathrm{T}]$;

Maiores detalhes sobre as equações diferenciais parciais e a aplicação do MEF para resolução de equações diferenciais podem ser encontrados em Anderson; Woessner (1992).

\section{Utilização da POO para Implementação do Simulador}

Para a implementação computacional do simulador foi utilizado o conceito de POO. Para este propósito, a linguagem Java da Sun Systems foi escolhida para programação e o ambiente Eclipse foi utilizado para a implementação dos códigos computacionais. A principal vantagem e motivo de escolha destas ferramentas foi o custo, já que o Java não é uma linguagem proprietária. $\mathrm{O}$ ambiente de programação Eclipse encontra-se disponível na WEB (www.eclipse.org) sem custos financeiros. Além disto, a adoção da linguagem Java permite também o uso de uma série de ferramentas, como a biblioteca Jakarta, que foi utilizada para resolver os sistemas de equações lineares. Ademais, o Java é uma linguagem multi-plataforma, de maneira que o produto final pode ser executado tanto no sistema operacional Windows ${ }^{\circledR}$ como no Linux.

Antes de detalhar o simulador, faz-se necessária a apresentação de alguns conceitos básicos da POO. Na POO, o desenvolvimento de um programa concentra-se na criação de classes, que são entidades abstratas representativas de elementos de um sistema real. As classes são formadas por atributos, que são os dados do sistema real, e métodos, que são suas funções. Um aqüífero é um exemplo de uma classe, que teria como atributos as condutividades hidráulicas nas duas direções, $\mathrm{X}$ e $\mathrm{Y}$, ressaltandose que o aplicativo desenvolvido não trabalha em 3 dimensões; já a simulação do fluxo subterrâneo seria um método dessa classe. Uma classe passa a ser objeto a partir do momento que ela é criada para ser usada num programa, ou seja, a criação de um ou mais aquífferos num programa a partir da classe aqüífero gerará objetos.

A POO conta com inúmeros conceitos que são intrínsecos a esta forma de programação, talvez os mais importantes e mais utilizados são: Generalização, que são classes que contém atributos e métodos gerais;

- Especialização, que ocorre no momento da criação de classes a partir de classes gerais. $\mathrm{Na}$ classe especializada são criados atributos e métodos específicos dessa classe;

- Herança, que está ligada aos dois últimos itens. Quando da criação de classes especializadas (subclasses) a partir de classes generalizadas (superclasses), todos os atributos e métodos da superclasse são herdados pela subclasse;

As características intrínsecas da $\mathrm{POO}$ conduzem a códigos mais simples, permitindo o reuso de classes e a criação de novas classes a partir de superclasses, evitando-se assim a repetição de códigos.

Como relação ao simulador implementado, um conjunto de classes e pacotes (agrupamento de classes por finalidade comum) foi idealizado. A figura 1 apresenta os pacotes que compõem o simulador e a sua classe principal (a GWSYSTEM). O pacote principal, que tem como finalidade principal a simulação do fluxo de águas subterrâneas, foi nomeado de groundwater, é neste pacote que a classe principal e os seis pacotes se encontram. O simulador do fluxo de águas subterrâneas conta com um total de 35 classes. 


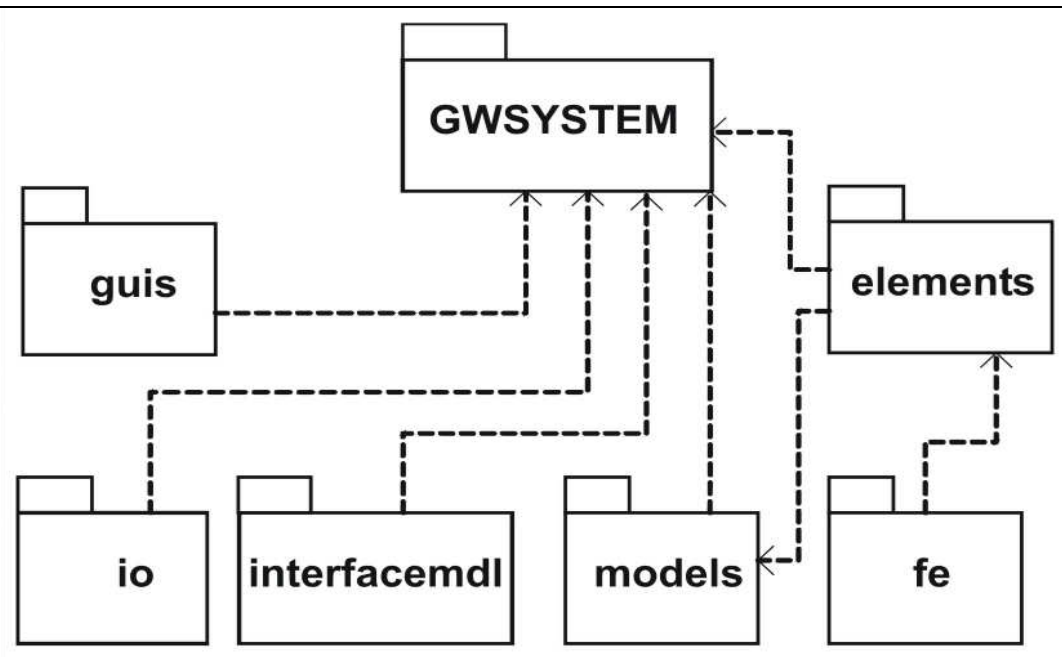

Figura 1- Pacotes e classe principal do simulador de fluxo de águas subterrâneas Figure 1-Packages and main class of the groundwater model

Os seis pacotes e a classe principal do simulador ilustrados na figura 1 são descritos a seguir:

- Pacote guis - As GUI's são as janelas de entrada, saída e manipulação de dados. Através delas, as propriedades de poços e aqüíferos podem ser acessadas, os procedimentos são executados e os resultados das simulações são disponibilizados;

- Pacote $f e$ - Composto por duas classes, a FELocalMat3x3 e FELocalVector. A primeira é utilizada para representar a matriz local, de dimensão três por três, de cada elemento triangular. A segunda auxilia no cálculo das matrizes locais;

- Pacote elements - Composto por sete classes responsáveis pela representação do sistema em simulação, aqüíferos, zonas de recargas e poços, e pela sua discretização em termos de elementos triangulares e nós, necessários ao MEF. Nele encontram-se as classes TriangleAtt, NodeAtt, Aquifer, BoundaryCondition, RechargeZone, Well e WellStepProfile;

- Pacote io - Responsável pela transferência de dados entre arquivos;

- Pacote interfacemdl-É o pacote responsável pela integração do SIG e do simulador. Através da classe GWS_STDSS são extraídos os atributos de uma camada no formato shape (*.shp), do ArcView ${ }^{\circledR}$, por exemplo. Com esses atributos são criados os objetos representativos do sistema a ser simulado, os quais são representados por entidades geométricas;

- Pacote models - Responsável pela construção das matrizes e da solução do sistema de equações. É nele que se encontram implementados os procedimentos numéricos do
MEF. Este pacote é formado por duas classes: a primeira (FEMeshModel), que tem a função de armazenar os elementos triangulares e seus nós; a segunda (FEIntegration) é responsável pela simulação dos dois tipos de problemas implementados;

- Classe GWSYSTEM - Esta classe encontra-se dentro do pacote geral, o groundwater. Ela concentra o acesso às demais classes do pacote computacional. É através dela, que as GUI's acessam as diversas classes dos seis pacotes aqui apresentados. É também nesta classe que os principais atributos do sistema (conceito similar à variável da programação estruturada) ficam armazenados.

- O pacote elements (Figura 1) é detalhando na figura 2, que pode ser divida em quatro colunas: As duas primeiras, da esquerda, descrevem o sistema a ser simulado, através das classes Aquifer (aquífero), Well (poço) e RechargeZone (zona de recarga). Já as duas colunas da direita são compostas por classes que descrevem o sistema em simulação através de TriangleAtt (triângulos), NodeAtt (nós) e BoundaryCondition (condições de contorno). Como se pode notar, todas as classes são criadas com base em classes geométricas. Esse padrão utilizado na criação dessas classes é típico da POO e é denominado de herança.

Cada classe desse pacote foi criada com base numa classe geométrica que a caracteriza no espaço, por exemplo, a classe poço foi criada com base numa classe que representa um ponto. $\mathrm{Na}$ superclasse ponto, só há atributos (x e y, por exemplo) e métodos (determine a distância a outro ponto, por exemplo) relativos à esta geometria. Já na classe poço, são declarados os 
atributos necessários à descrição dessa entidade id (código), name (nome), constFlow (vazão), etc., e seus métodos (leia o nome (getName), o código (getID), a vazão (getFlow), etc.). Outra classe ilustrada na figura 2 é a TriangleAtt, que possui atributos como o aqüífero, um identificador e uma zona de recarga. Seus métodos, por exemplo, o $\operatorname{get} K x()$, acessam a classe representativa do aquiífero. Um triângulo não tem $\mathrm{K}_{\mathrm{x}}$ (condutividade hidráulica) como seu atributo, porém essa propriedade encontra-se no atributo aquifer. Através dos métodos getMatrixCLocal() e getMatrixKLocal() são construídas as matrizes 3 por 3 que agregam as propriedades geométricas do triângulo e os atributos hidráulicos do aqüífero. A classe TriangleAtt foi criada com base na superclasse GeoTriangle2D, representativa de um triângulo. Assim, métodos que dizem respeito à geometria do triângulo são herdados dessa superclasse. Caso o usuário deseje ter acesso a informações sobre os nós que compõem esse triângulo pode fazê-lo através do getNodeI(),getNodeJ() e getNodeK().

Outro motivo para a criação das classes que representam o sistema a partir das classes geométricas é a integração do simulador ao SIG. Dessa foram, a geometria representativa é obtida das entidades geométricas do SIG.

Ainda com relação à figura 2 , as linhas cheias existentes entre as classes apresentadas nesta figura indicam seus relacionamentos. Estes relacionamentos procuram mostrar como as classes são compostas, por exemplo, entre a classe TriangleAtt e NodeAtt há um relacionamento de 1 para 3 , indicando que cada TriangleAtt é composto de três NodeAtt. São através destes relacionamentos que atributos de uma classe ou métodos podem ser acessados.

\section{Integração do Simulador de Águas Subterrâneas ao SIG}

O SIG utilizado para compor o ARENA foi o JUMP (Unified Mapping Platform). O JUMP (2003) é um aplicativo de código aberto, que foi desenvolvido pela Vivid Solutions $\odot$, empresa canadense. Entre as vantagens que este
SIG apresenta, três merecem destaque. Primeiro, aimplementação computacional através dos conceitos da POO, em linguagem Java, facilita o entendimento de seu código computacional e permite a execução do mesmo em diferentes plataformas. Segundo, a questão da implementação de seus códigos computacionais, baseados em normas e padrões internacionais do consórcio Open Geospatial Consortium - OGC (www.opengeospatial.org), também facilita $\mathrm{O}$ acesso a seus códigos computacionais. E a última diz respeito aos custos financeiros, pois este SIG encontra-se disponível gratuitamente na WEB.

O consórcio OGC, que vem criando normas e padrões para a área de geotecnologias, conta com uma norma que padroniza as camadas de informação (layers), denominadas de features. É nas features que a geometria e os dados tabulares relacionados à geometria são armazenados. Elas também guardam informações sobre o estado das camadas, cores, espessuras de linhas, etc. Sua utilização direta implicaria no carregamento destas informações também, as quais não são necessárias a simulações. Assim, optou-se pela criação de um pacote representativo das camadas de informação (layer), porém contendo apenas as entidades geométricas e as informações relacionadas a cada objeto geométrico. Dessa forma, sem depender das características específicas do JUMP, o simulador pode ser integrado a qualquer outro SIG através da interface desenvolvida.

A figura 3 ilustra o esquema utilizado para a integração das entidades geométricas do JUMP com as classes do simulador.

O processo se resume ao seguinte: uma camada de informações carregada no JUMP é enviada para a classe principal do pacote groundwater, a GWSYSTEM. Esta classe envia a camada para o pacote interfacemdl (Figura 1), que filtra a camada de informações, retornando à classe principal objetos do pacote Feature Set (Figura 3). Estes objetos por sua vez são utilizados para criar os objetos representativos do sistema em simulação, que advêm do pacote elements (Figura 1). 


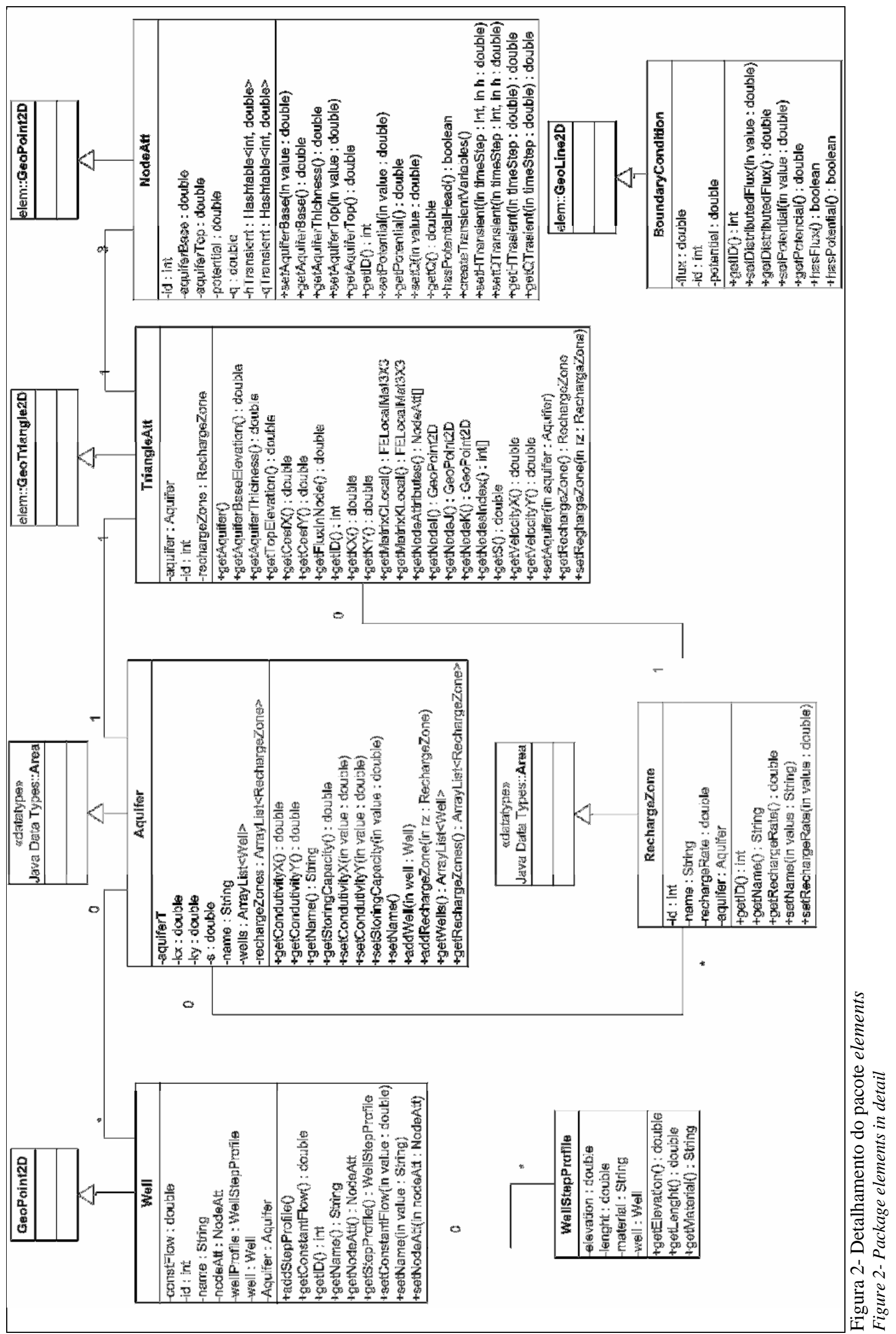




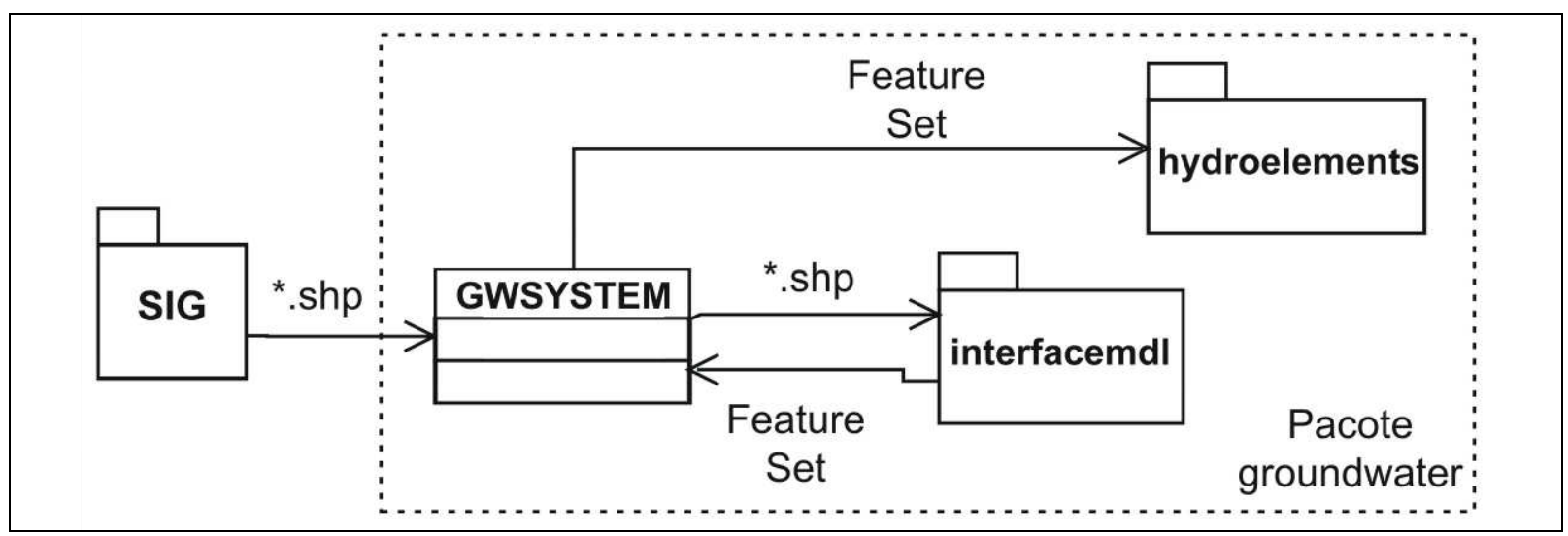

Figura 3- Esquema de integração do SIG ao simulador Figure 3-Integration of the GIS to the model

\section{APLICAÇÃO DO ARENA A UM SISTEMA HIPOTÉTICO}

A fim de testar o ARENA, o simulador e sua integração ao JUMP, procedeu-se à modelagem de um sistema hipotético. Este sistema representa um aquífero confinado e sua simulação foi realizada para o regime transiente. $\mathrm{O}$ mesmo foi tomado como base para validação do simulador programado e foi extraído do livro de Kinzelbach (1995). Este exemplo foi utilizado para validar o modelo programado, uma vez que suas soluções podem ser obtidas analiticamente e por meio do programa constante em Kinzelbach (1995). A figura 4 ilustra o sistema com a rede de elementos finitos utilizada para a simulação.

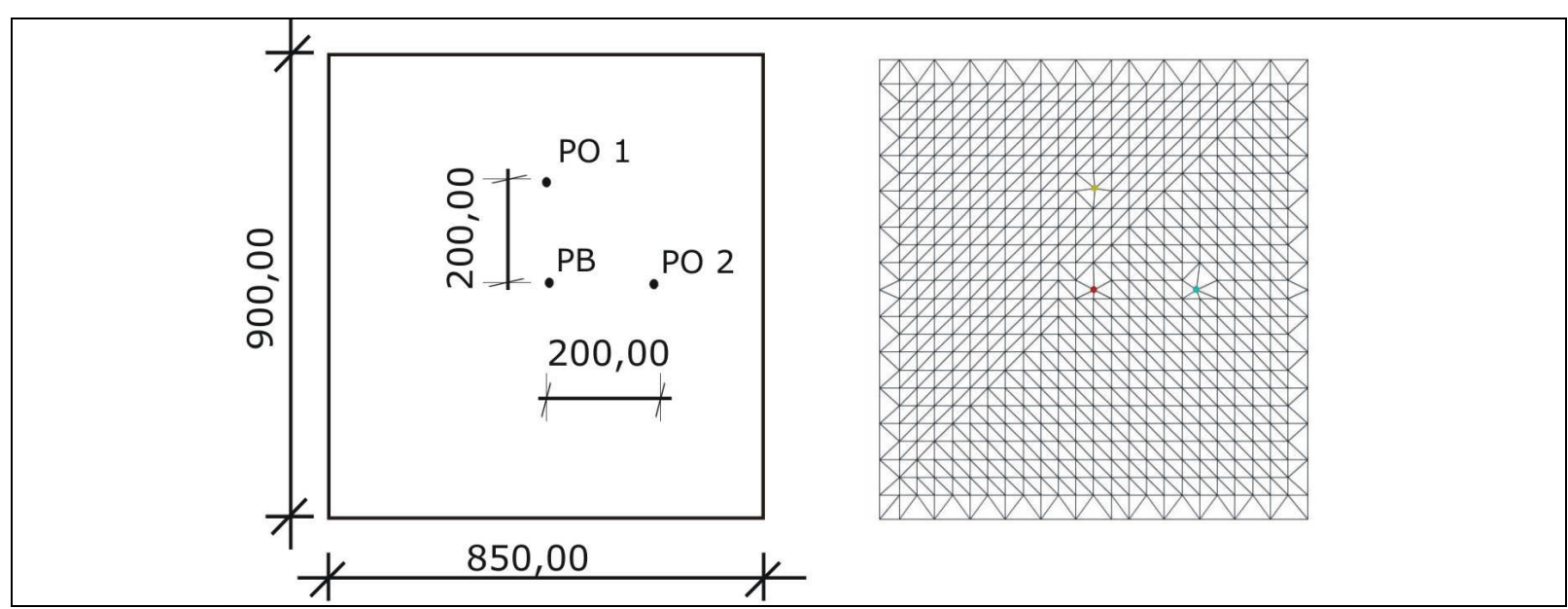

Figura 4- Sistema hipotético com a rede de elementos finitos utilizada para a simulação

Figure 4- Hypothetic system with Finite Element network used for the simulation

O sistema ilustrado na figura 4 apresenta três poços, um localizado no centro do sistema (PB), bombeando $0,05 \mathrm{~m}^{3} / \mathrm{s}$, um localizado ao lado direito do poço de extração (PO 2), mais precisamente a $200,00 \mathrm{~m}$ desse poço, e outro na parte superior (PO 1), também a 200,00 $\mathrm{m}$ do poço de referência. Este sistema conta ainda com as seguintes características:

\section{Hidráulicas e físicas:}

- Condutividade hidráulica (K): 0,0005 m/s;

- Coeficiente de armazenamento (S): 0,001;

- Espessura do aqüífero (b): 20,00 m;

\section{Condições de contorno:}

- Nos lados superior e inferior tem-se o fluxo conhecido, constante e igual a zero e a carga hidráulica desconhecida;

- Nos lados esquerdo e direito tem-se a carga hidráulica conhecida, constante e igual a 50,00 m e o fluxo desconhecido;

\section{Condição inicial:}

- Inicialmente, carga hidráulica é constante e igual a 50,00 m em todos os pontos do aqüífero;

A simulação foi realizada para intervalo de tempo $\Delta t=1 \mathrm{~min}$ até um total de 14.400 minutos, 
ou seja, um dia. A figura 5 apresenta a variação temporal da carga hidráulica nos três poços existentes no sistema. Esse resultado confronta os resultados obtidos pelo programa desenvolvido (linhas cheias da legenda) e os resultados do programa ASM - Aquifer Simulation Model (KINZELBACH, 1995) (pontos da legenda), baseado no MDF para resolução das equações diferenciais parciais. A figura 5 mostra, ainda, que variações mais bruscas da carga hidráulica ocorrem até as três primeiras horas de bombeamento, principalmente no poço que há o bombeamento e que o sistema tende a entrar em equilíbrio a partir de aproximadamente 800 minutos (13 horas) do início da operação. As diferenças encontradas entre as curvas de rebaixamento observadas na figura 5 devem-se à dois fatores: primeiro, à discretização realizada no ARENA o delta $\mathrm{x}$ utilizado foi equivalente a 1/3 do ASM, ou seja, mais detalhada; segundo à discretização temporal, no ARENA a simulação foi realizada para intervalos de $1 \mathrm{em} 1$ minuto. Salienta-se que a forma de discretização do aplicativo programado é através de triângulos, enquanto que do programa ASM e quadriláteros, não sendo possível assim discretizações semelhantes.
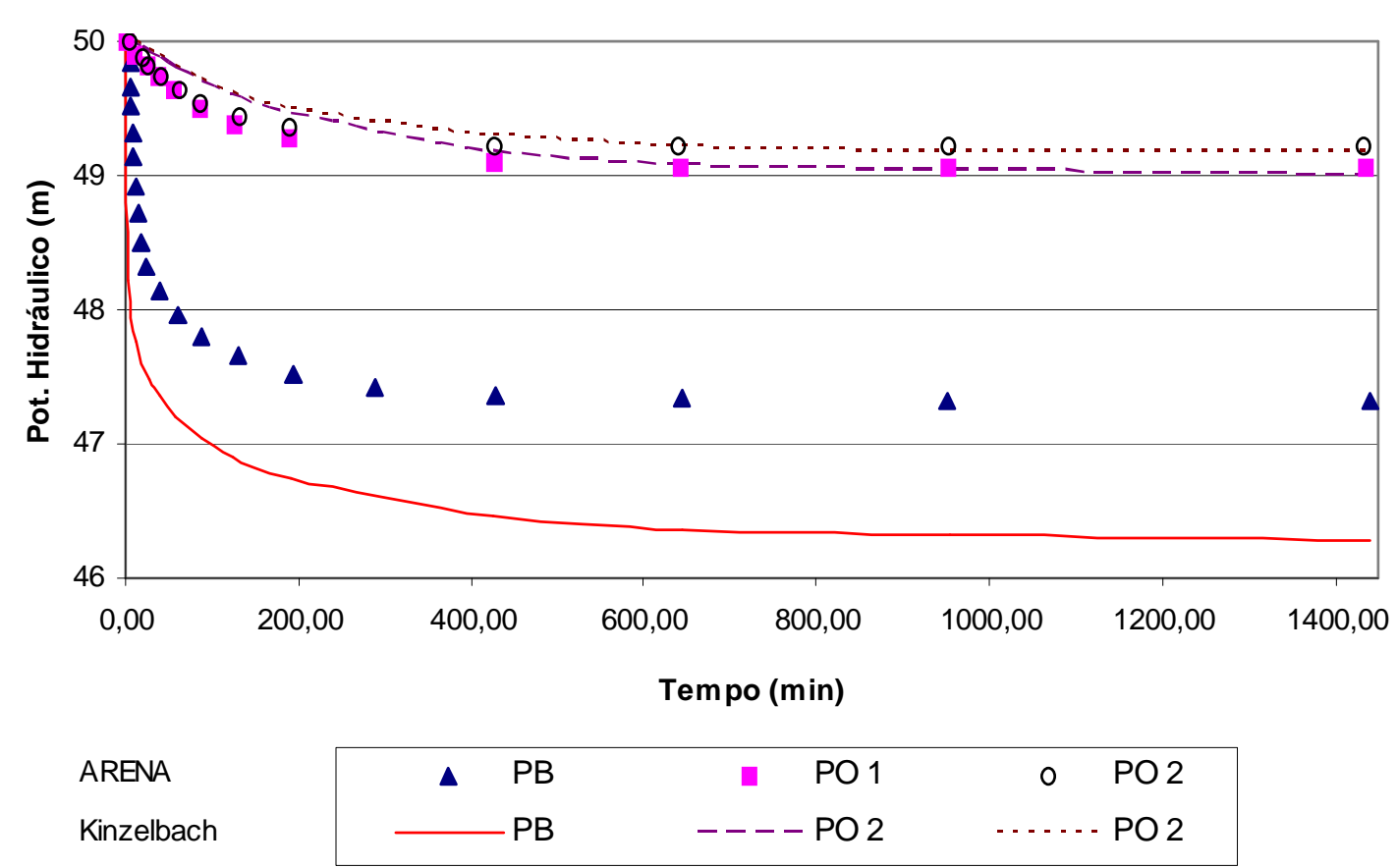

Figura 5- Variação da carga hidráulica ao longo de um dia (poço de bombeamento e poços de observação) Figure 5- Hydraulic head variation during a day (pumping well and monitoring wells)

Como se pode observar nas curvas apresentadas na figura 5 as simulações apresentam resultados semelhantes. As diferenças numéricas existentes devem-se a aplicações de métodos numéricos diferentes às formas de discretização utilizadas pelo diferentes métodos numéricos.

Uma vez validado o simulador, foi utilizado o JUMP para visualização dos resultados. Na figura 6 é apresentada a distribuição de cargas hidráulicas simuladas, após três horas de bombeamento com uma vazão de $0,05 \mathrm{~m}^{3} / \mathrm{s}$ no poço localizado no centro do sistema. Utilizou-se uma escala de cores cinza, onde as cores mais escuras representam as maiores cargas hidráulicas. A legenda apresentada ao lado direito da figura mostra os valores mínimos dos intervalos de agrupamento das cargas hidráulicas.

A figura 7 apresenta a variação temporal das cargas hidráulicas numa seção transversal que passa pelo poço de bombeamento. Nela pode-se acompanhar o cone de rebaixamento, que ao final das 24 horas de simulação atingiu 3,88 m no poço de bombeamento.

O JUMP não conta com uma ferramenta para traçado de isolinhas, portanto o traçado de equipotenciais não é possível. Porém, foi programada uma rotina de exportação de resultados que permite a criação de um arquivo texto, compatível com formato de programas de visualização. 


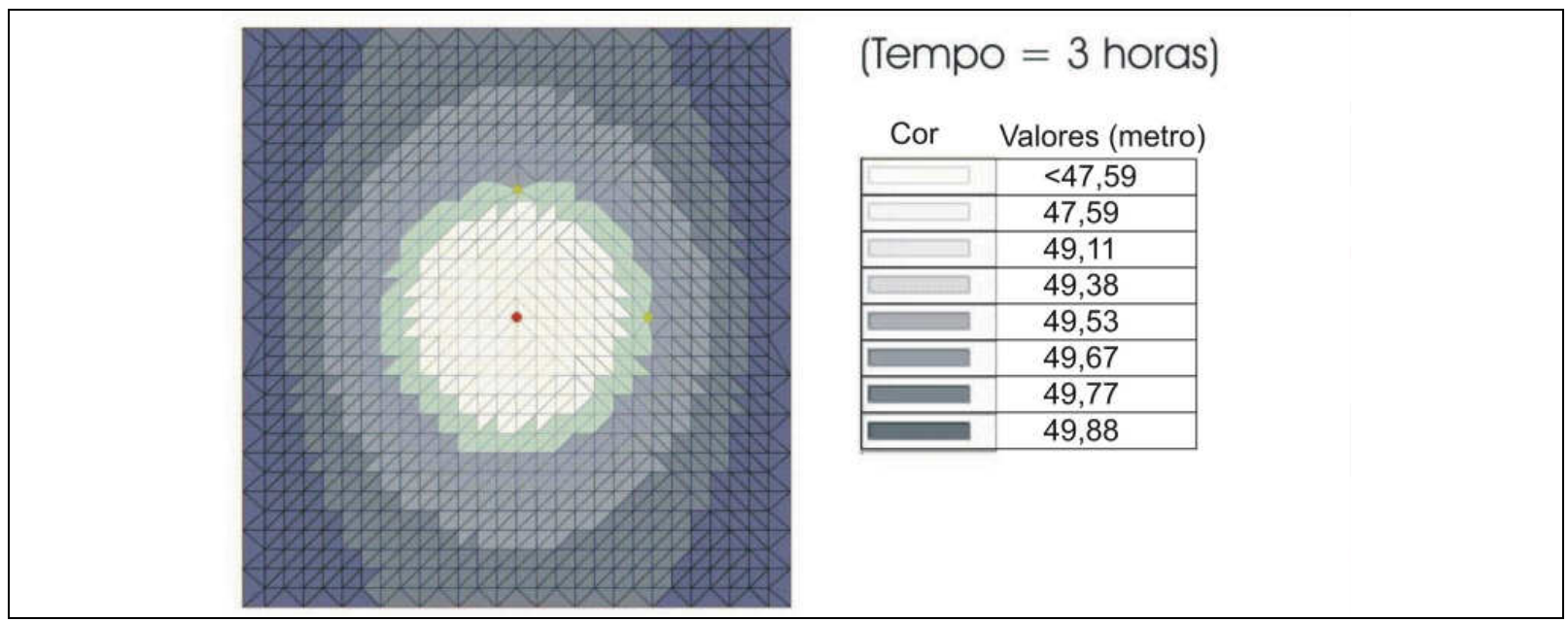

Figura 6- Distribuição da carga hidráulica no domínio estudado (tempo = 3 horas)

Figure 6- Hydraulic head distribution for the studied system (time step $=3$ hours)

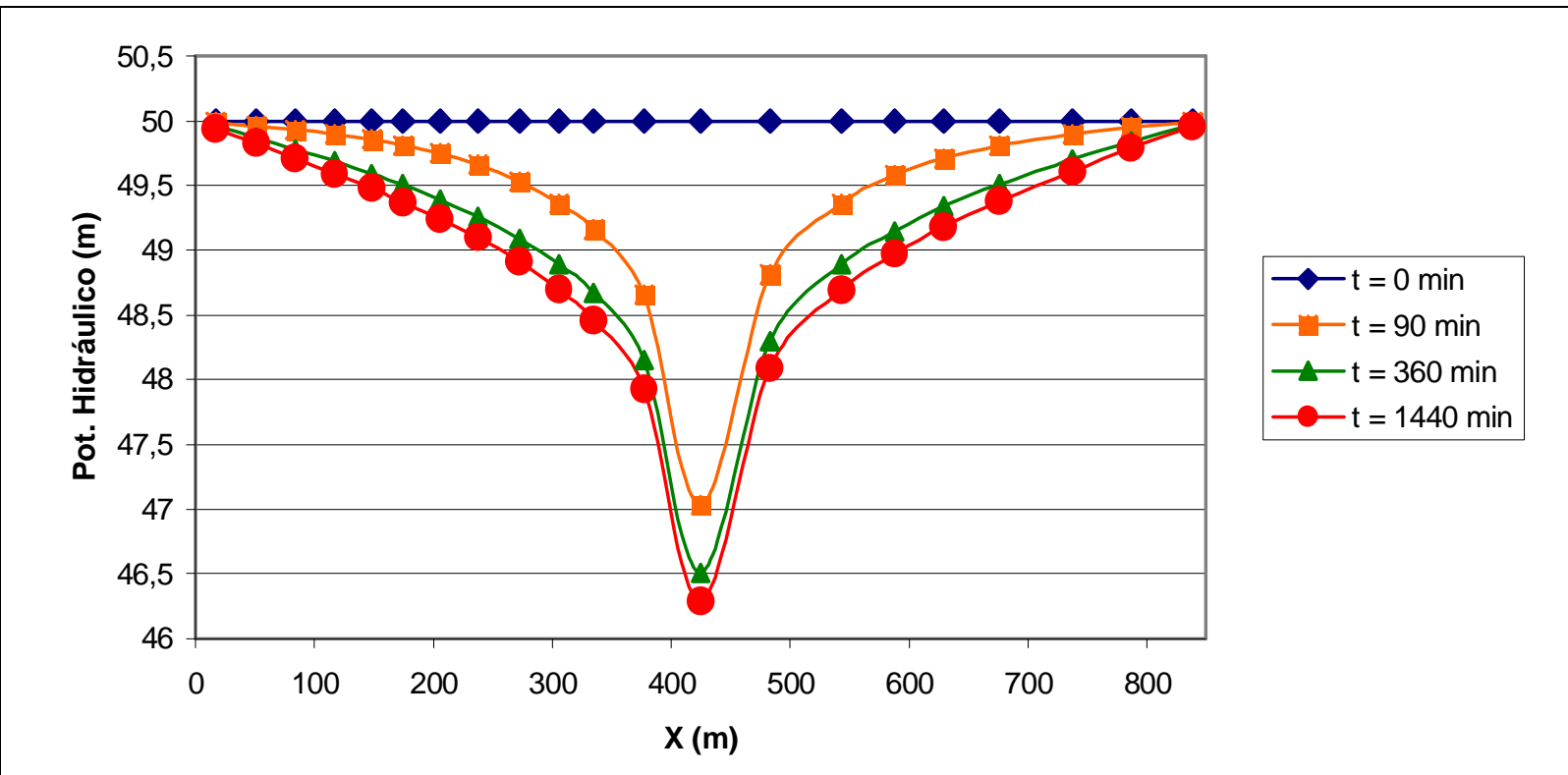

Figura 7- Variação temporal das cargas hidráulicas na seção transversal que passa pelo poço de extração Figure 7- Hydraulic heads variation for a section through the pumping well

\section{CONCLUSÕES}

Neste artigo, um simulador do fluxo de águas subterrâneas (baseado no Método de Elementos Finitos) foi apresentado. O simulador foi integrado a um Sistema de Informações Geográficas (SIG), o JUMP, compondo assim um Sistema de Suporte à Decisão (SSD). O SSD foi aplicado a um sistema hipotético em regime transiente, a fim de validar o simulador. No decorrer do cumprimento dessas etapas do trabalho, as seguintes observações e conclusões puderam ser verificadas:

- A programação orientada a objetos (POO) permitiu um melhor entendimento do sistema hipotético simulado, facilitando assim o desenvolvimento do programa e as futuras modificações e manutenções desse programa;

- As características intrínsecas da POO (herança, generalização, especialização, etc.) facilitaram o processo de integração simulador/SIG;

- A utilização conjunta de técnicas como POO e UML permite um melhor entendimento dos sistemas, facilita a transferência de informações entre usuários, o armazenamento de dados, além de melhorar a compreensão dos pacotes, classes e, conseqüentemente, do programa como um todo;

- O simulador implementado modelou corretamente o sistema hipotético estudado, de forma que ele pode ser considerado validado, por comparação com resultados apresentados na literatura;

- A utilização de um SIG no apoio ao processo de modelagem é adequada, visto que no ARENA os dados necessários às simulações podem ser melhores sistematizados; 
- O SIG utilizado é promissor, porém faz-se necessária a implementação de pacotes para interpolação de valores, traçado de isolinhas e traçado de superfícies, para melhorar a visualização dos resultados de simulações do fluxo de águas subterrâneas;

- O JUMP necessita ainda da implementação de um pacote para impressão de resultados, o que melhorará a saída de dados deste SIG;

Por fim, salientam-se as propriedades do SSD como um todo que, devido ao ambiente de desenvolvimento (Eclipse), à linguagem de programação (Java) e ao SIG (JUMP) utilizados, gerou um programa isento de custos financeiros relacionados à aquisição desses aplicativos. Para o desenvolvimento de um sistema, que utilize soluções semelhantes, faz-se necessária apenas mão-de-obra especializada. Em, geral, soluções que utilizam linguagens, SIG's comerciais e sistemas proprietários elevam os custos do produto final, chegando muitas vezes a inviabilizar determinadas soluções. Desta forma, recomenda-se o uso de ferramentas semelhantes para o desenvolvimento de SSD's e aplicativos para a área de recursos hídricos.

\section{AGRADECIMENTOS}

Agradeço aos professores Alain Marie Bernard Passerat de Silans e Tarciso da Silva Cabral pelo apoio para desenvolvimento deste trabalho. Agradeço ainda ao ITT (Institut für Tropentechnologie) da Faculdade de Ciências Aplicadas de Colônia pelo apoio logístico durante minha estada na Alemanha. Por fim, externo agradecimentos ao $\mathrm{CNPq}$, DAAD e CAPES pelo apoio financeiro para desenvolvimento do trabalho de doutorado. 


\section{REFERÊNCIAS}

ALMEIDA, C.N. Implantação de um sistema de apoio ao planejamento e gerenciamento de recursos hídricos na bacia do rio do Peixe, com ênfase ao modelo de transformação chuva-vazão. 2001. 151 p. Dissertação (Mestrado) - Escola de Engenharia de São Carlos, Universidade de São Paulo, São Paulo, 2001.

ANDERSON, M.P.; WOESSNER, W.W. Applied groundwater modeling-simulation of flow and advective transport: San Diego: Academic Press, Inc., 381p. 1992.

ASSIS, F. Aplicação de um Sistema de Suporte à Decisão à Alocação de Água: o SSD da COGERHCE. In:SIMPÓSIO BRASILEIRO DE RECURSOS HÍDRICOS, 13., 1999, Belo Horizonte, 1999.

BABAIE, H.A.; BABAIE, A. Modeling geological objects with the XML Schema. Computers \& Geosciences, v.31, n.9, p.1135-1150, 2005.

BEVEN, K.J.; KIRKBY, M.J. A physically based, variable contributing area model of basin hydrology. Hydrological Sciences Bulletin, n.24, p.24-69, 1979.

CAVALCANTI, M.A.M.P. A modelagem matemática associada ao sistema de informações geográfica como instrumento de previsão no estudo do impacto hidrogeológico de reservatórios, 2002. 188p. Tese (Doutorado em Recursos Minerais e Hidrogeologia), Instituto de Geociências, Universidade de São Paulo, São Paulo, 2002.

CHAPRA, S.; CANALE, R.P., Numerical Methods for Engineers: With Software and Programming Applications, McGraw-Hill Publishing Co., 2002.

DIERSCH, H.J. Interactive, graphics-based finiteelement simulation system FEFLOW for modeling groundwater flow, contaminant mass and heat transport processes. FEFLOW User's Manual Version 4.5, 1996.

FILHO, J.S.V.; LANNA, A.E.L. O Paradigma da Modelagem Orientada a Objetos Aplicada a Sistemas de Recursos Hídricos - (I) Modelo Básico de Objetos para um Rede Hidrográfica, Revista Brasileira de Recursos Hídricos, v.8, n.8, p.79-89, 2003.

JUMP Unified Mapping Platform - Technical Report.
Disponível em: 〈http://www.vividsolutions.com/> Acesso em: março de 2005.

KINZELBACH, W.; RAUSCH, R. Grundwassermodellierung - Eine Einfuehrung mit Übungen. Gebrüder Borntraeger Berlin-Stuttgart, 1995.

KYRILLOS, D.S.; CIRILO. J.A. SIGMA - Sistema de informações para gestão do meio ambiente. Estudo de caso: Bacia do Rio São Francisco. In: SIMPÓSIO DE RECURSOS HÍDRICOS DO NORDESTE, 5., 2000, Natal, RN, 2000.

LAL, A.M.W.; ZEE, R.V.; BELNAP, M. Case Study: Model to Simulate Regional Flow in South Florida. Journal of Hydraulic Engineering, v.131, n.4, p.247-258, 2005.

MCDONALD, M.G.; HARBAUGH, A.W. A modular three-dimensional finite-difference ground-water flow model, USGS TWRI Chapter 6A1, 586p. 1988.

PORTO, R.L.; AZEVEDO, L.G. Sistemas de suporte a decisões aplicados a problemas de recursos hídricos. Técnicas quantitativas para o gerenciamento de recursos hídricos. Coordenação e organização científica: PORTO, R.L. Porto Alegre: Ed. Da Universidade/UFSGS/ABRH, (Coleção ABRH de recursos hídricos). p.42-95, 1997.

RODRIGUES, R.B. SSD RB - Sistema de Suporte à Decisão Proposto para a Gestão QualiQuantitativa dos Processos de Outorga e Cobrança pelo Uso da Água, 2005. 179p. Tese (Doutorado em Hidráulica e Saneamento)-. Escola Politécnica, Universidade de São Paulo, São Paulo, 2005

TSOU, M.S.; WHITTEMORE, D.O. User Interface for Ground-water Modeling: ArcView Extension. Journal of Hydrologic Engineering, v.6, n.3, p.251257, 2001.

WANG, J.; HASSET, J.M.; ENDRENY, T.A. An object oriented approach to the description and simulation of watershed scale hydrologic processes. Computers \& Geosciences, v.31, n.4, p.425-435, 2005. 
Desenvolvimento e integração de um simulador de fluxo subterrâneo a um sistema de informaçães geográficas 